\title{
Did This Patient Have Lumbar Fusion? Review of MRI Findings
}

\author{
Zacharia Isaac, $M^{\star}$, Evan Siegelman, $M^{\star \star}$ and Laurie A. Loevner, MD*
}

\section{CASE PRESENTATION}

A 69-year old man presents with complaints of low back pain and bilateral lower extremity pain. He has a history of multiple spine surgeries. In 1981, he underwent a L4/5 discectomy without relief of symptoms. Later, in 1981, he underwent an L5/S1 discectomy, which also failed to alleviate his symptoms. In 1993, he underwent a posterior fusion surgery with pedicle screws, which brought him no pain relief. In 1995, he underwent removal of hardware and a posterolateral fusion with an iliac crest bone graft. This also failed to bring him relief of his pain. In 1996, a spinal cord stimulator was placed and subsequently removed in 1997. This also failed to bring him lasting relief. At no time did he undergo an interbody fusion. An MRI was obtained (Figs. 1-6). What is the differential diagnosis?

\section{DISCUSSION}

The MRI of the lumbar spine demonstrates significant marrow changes in the vertebral bodies of L 3 and L4 with complete collapse of the L3-4 intervertebral disc (Figs. 1 and 2). The T1 and T2 weighted axial image demonstrates increased signal in the vertebral body, posterior elements and moderate central stenosis (Figs. 4 and 5). There is no enhancement with the administration of gadolinium contrast (Figs. 3 and 6). There exists a broad differential diagnosis for changes in bone marrow appearance adjacent to the vertebral end plates and destruction of the intervertebral disc, which includes degenerative changes, malignancy, osteomyelitis, discitis, and postoperative

From Hospital of the University of Pennsylvania, Philadelphia, Pennsylvania. *Dr. Isaac is a fellow at Penn Spine Center, Department of Rehabilitation Medicine, ** Dr. Siegelman is associate professor of the Department of Radiology and "Dr. Loevner is associate professor of the Department of Radiology at the Hospital of the University of Pennsylvania. Address correspondence: Zacharia Isaac, MD, 3400 Spruce Street, Philadelphia, PA 19104. E-mail: zisaac@yahoo.com changes. Given that there is no history of trauma, infection, malignancy, constitutional symptoms, or interbody fusion, the appearance of the L3 and L4 vertebral bodies and the collapse of the L3-4 intervertebral disc pose a diagnostic dilemma.

Modic et al (1) described a classification scheme for endplate changes associated with degenerative disc disease. Type I changes are characterized by decreased signal on T1 and increased signal on T2 weighted images. Histological specimens reveal fissuring and disruption of the vertebral endplates with vascularized fibrous tissue within the adjacent marrow. Type II changes are characterized by increased signal on $\mathrm{T} 1$ and slightly increased or isointense to bone marrow on $\mathrm{T} 2$ sequences. Histology reveals replacement by fatty yellow marrow. Type III changes are characterized by decreased signal on $\mathrm{T} 1$ and T2 weighted sequences. Dense bony sclerosis occurs and the marrow fat is replaced by bone. This patient's MRI abnormalities are most consistent with Modic type II changes. Although these abnormalities are possibly related to a solely degenerative process, the MRI demonstrates disproportionate loss of disc height at L3-4 compared to the L4/5 and L5/S1 discs which more commonly undergo degenerative changes first (Fig. 2).

One should consider malignancy when evaluating elderly patients even in the absence of constitutional symptoms. Malignant neoplasms include primary bone tumors, hematologic malignancy, and more commonly metastatic disease. Malignancy can produce several patterns on MRI depending on the underlying malignancy. Type 1 lesions are focal and produce low signal intensity on $\mathrm{T} 1$ and high signal intensity on $\mathrm{T} 2$ weighted sequences. This is seen with lytic or destructive metastasis such as is seen in multiple myeloma. Type 2 occurs with blastic or sclerotic lesions and focal areas of low intensity as seen on $\mathrm{T} 1$ and T2 weighted sequences. Type 3 changes produces inhomogeneous signal in the vertebral body. Type 4 changes produce homogenous decreased signal intensity on $\mathrm{T} 1$ and homogenous increased intensity on $\mathrm{T} 2$ weighted sequences. These patterns are seen in leukemia, lymphoma, and other infiltrative marrow processes. 


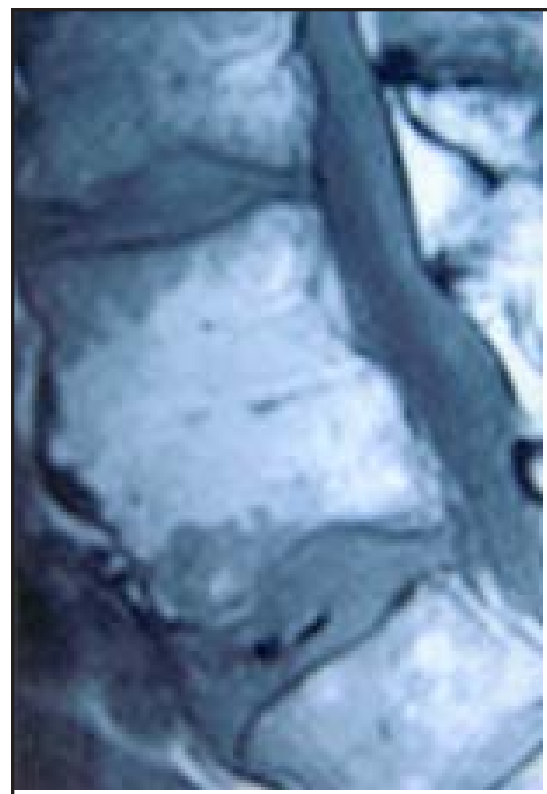

Fig. 1. T1 weighted, sagittal image demonstrating increased signal intensity of the L3 and L4 vertebral bodies adjacent to the collapsed L3-4 disc. There is vacuum disc phenomenon in the L2-3 and L4-5 disc with moderate decrease in height.

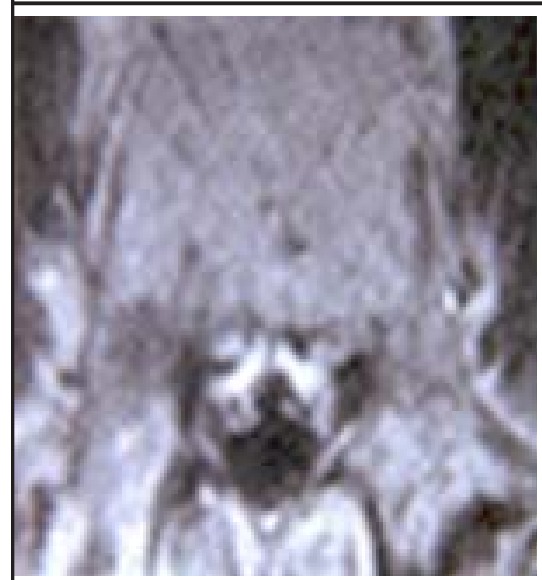

Fig. 4. T1 weighted, axial image demonstrating increased signal with sharply demarcated borders throughout the vertebral body, pedicle, and superior and inferior articular processes. There is moderate central stenosis.

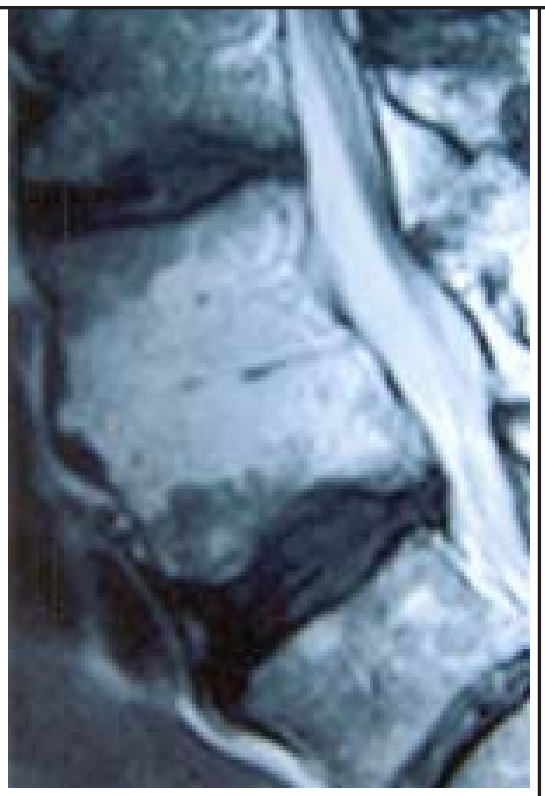

Fig. 2. T2 weighted, sagittal image demonstrating increased signal intensity of the L3 and L4 vertebral bodies adjacent to the collapsed L3-4 disc. There is vacuum disc phenomenon in the L2-3 and L4-5 disc with moderate decrease in height.

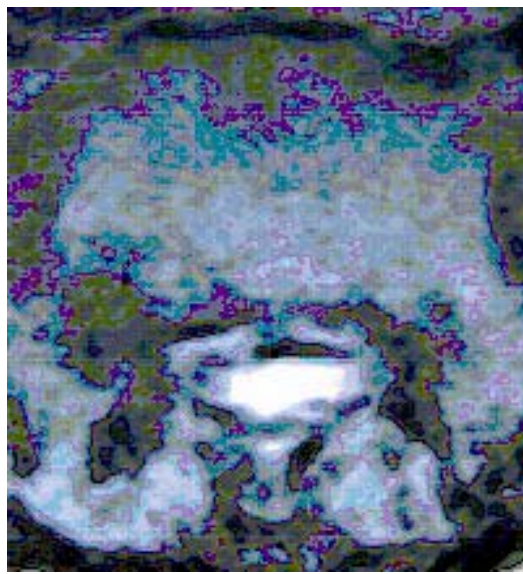

Fig. 5. T2 weighted, axial image demonstrating increased signal diffusely through the vertebral body, pedicle, and superior and inferior articular processes. There is moderate central stenosis.

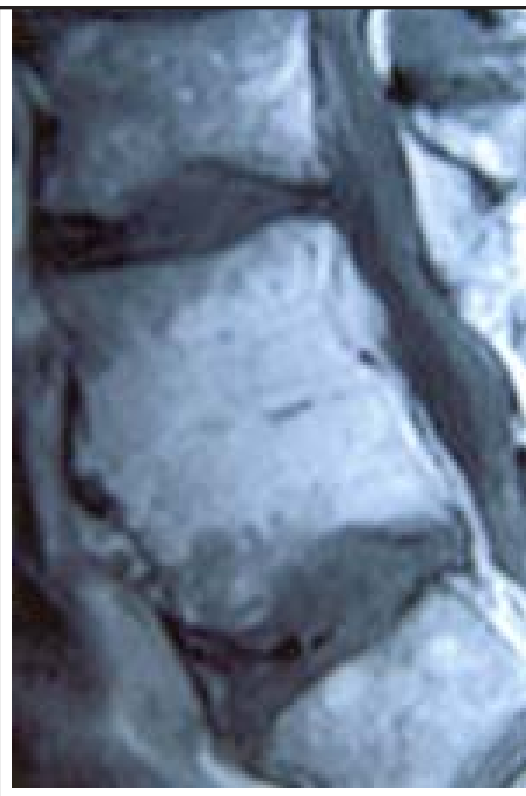

Fig. 3. T1 weighted, post gadolinium contrast, sagittal image shows no areas of new focal enhancement compared to without contrast (figure 1)

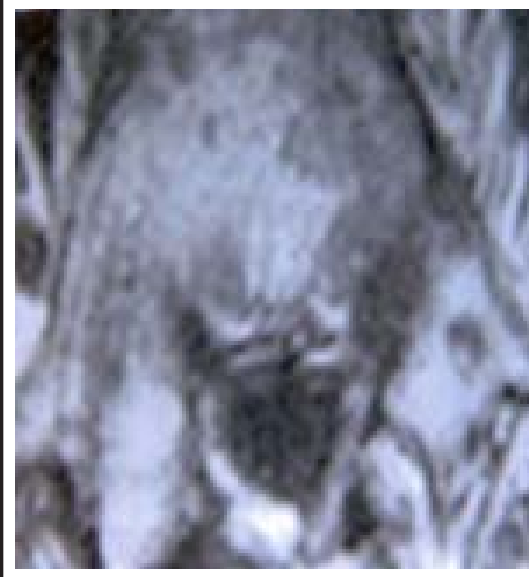

Fig. 6. T1 weighted, post gadolinium contrast, axial image shows no areas of increased uptake compared to pre contrast.(see figure 4)
MRI imaging sequences that are useful for identifying malignancy include short $\mathrm{T} 1$ inversion recovery sequences (STIR) and fat suppression images $(2,3)$. The use of gadolinium contrast is helpful in the diagnosis of malignancy. Gadolinium is a paramagnetic ion chelate that itself is not imaged by MRI. These paramagnetic agents bind to water and alter the molecular environment of the tissues in which it accumulates. These areas of accumulation are seen as areas of increased signal in the T1 weighted images. MRI has been demonstrated to have higher sensitivity than bone scintigraphy in suspected myeloma, renal cell carcinoma, and melanoma (4). Given 
our patient's long-standing history of back pain, lack of constitutional symptoms, and non-corroborative MRI findings, malignancy is unlikely. The patient's MRI shows increased rather than decreased signal intensity on the T1 weighted images, there is no bony destruction, and there is prominent disc involvement, which is not a typical feature of a malignant process.

MRI is useful for the diagnosis of nonmalignant tumors of the spine. Although the patient's MRI findings are not typical of these conditions, for instructional purposes, these conditions are briefly reviewed. Hemangiomas are found in $11 \%$ of persons $(5)$, are usually asymptomatic $(6,7)$, and are the most common benign tumors of the spine. They appear as well circumscribed areas of high signal intensity on $\mathrm{T} 1$ due to their adipose content and on $\mathrm{T} 2$ due to their angiomatous component. This often serves to distinguish it from fat, which can demonstrate decreased signal on T2 weighted images. Osteoid osteomas, most commonly found in males between 5 and 20 years of age, most commonly arise in the lumbar spine and its posterior elements $(8,9)$. It demonstrates low signal intensity on $\mathrm{T} 1$ and $\mathrm{T} 2$ weighted images, and plain radiograph correlated images show surrounding sclerosis. When the nidus is larger than $1 \mathrm{~cm}$ to $1.5 \mathrm{~cm}$, it is classified as a giant osteoid osteoma. Eosinophilic granuloma, associated with Langerhans cell histiocytosis, or Histiocytosis X can cause single or multiple infiltrating bony granulomas of low signal intensity on T1 and high signal intensity on T2 weighted images. Our patient's predominant findings involved collapse of the intervertebral disc with surrounding endplate marrow changes. Since these features are not characteristic of these conditions, it is unlikely that these diagnoses could account for the patient's findings.

Since our patient's MRI abnormalities have sharply demarcated borders and involve predominantly the vertebral endplates, it is unlikely that the patient has a disease of marrow infiltration, however, it is important to briefly consider this possibility. The vertebral body acts as a major hematopoietic center, and with age the red marrow undergoes transformation to fatty yellow marrow. With aging, linear and triangular areas of fatty deposition occur along the endplates (10). Bone marrow disorders are classified into 4 main categories: reconversion (hyperplastic anemias), infiltration (tumors, infection, storage disorders, myeloproliferative disorders), ischemia or edema (avascular necrosis, complex regional pain syndrome, osteoporosis), and depletion (aplastic anemia, radiation therapy, chemotherapy) (11-13). Accurate differentiation based on MRI appearance alone is difficult. Corroborative diagnostic testing and clinical correlation are critical for obtaining accurate diagnosis.

Infection is a diagnostic possibility, although history and examination do not support this diagnosis. The intervertebral disc is a relatively avascular structure, and its involvement by a pyogenic infectious process is via hematogenous spread to the adjacent vertebral bodies and subsequent direct invasion of the intervertebral disc. Exceptions to this include iatrogenic causes where the disc may be infected first by cutaneous skin flora post procedurally. The lack of vascular blood flow to the intervertebral disc limits the effectiveness of the immune response and this leads to rapid destruction of the intervertebral disc. The adjacent endplates are quickly invaded and adjacent vertebral bodies can be affected by osteomyelitis. If the epidural space is involved, infection can rapidly spread beyond the adjacent levels. MRI can demonstrate abnormalities earlier than CT or plain radiographs $(14,15)$. T1 weighted images demonstrate decreased signal in the vertebral bodies and involved disc, and absent or poorly demarcated cortical margins. T2 weighted spin-echo sequences demonstrate increased signal intensity in the disc and adjacent endplates. Gadolinium enhanced images will also show increased uptake in these anatomic locations as well. The posterior elements are often spared. There is loss of disc space height and varying degrees of bony destruction depending on the degree of osteomyelitis. STIR and T1 weighted images with fat suppression are often helpful in identifying infected tissue (16). Bone erosion is best depicted on proton density images or T1 weighted fat suppression sequences. Tuberculosis most often involves the thoracic vertebral bodies (Pott's disease), however, its clinical presentation and MRI appearance differ from that of pyogenic organisms. It has an insidious onset with symptoms progressing over months to years. There is widespread destruction of the vertebral bodies with relative preservation of the disc spaces. The anterior portion of the vertebrae is affected resulting in anterior compression and a kyphotic gibbous deformity. Skip lesions are seen as the infection spread beneath the anterior longitudinal ligament to nonadjacent vertebrae. Our patient, in contrast, has disc space collapse without significant bony destruction, making this diagnosis unlikely.

MRI is useful in the evaluation of patients with persistent pain post surgery. Common etiologies of the failed back surgery syndrome include spinal stenosis from foraminal, lateral, or central stenosis, internal disc disruption 
syndrome, epidural fibrosis, recurrent or retained herniated disc, and mechanical low back pain secondary to degenerative disc disease (17). MRI can be helpful in the diagnosis of several of these diagnoses. Recurrent or retained herniations exhibit decreased signal intensity on T1 and T2 weighted images. Post gadolinium contrast MRI can differentiate recurrent disc from epidural scarring $(18,19)$. Since scar tissue receives vascular supply and herniated discs do not, scar demonstrates increased signal intensity on T1 weighted post-contrast images.

Although one cannot be certain of what the underlying diagnosis is, a hypothesized explanation of the MRI appearance of the L3-4 segment is accelerated degenerative disc disease due to prior instability with subsequent autofusion. The vertebral marrow near the endplates has been replaced by fat as part of the degenerative process (Figs. 1 and 2).

\section{REFERENCES}

1. Modic MT, Steinberg PM, Ross JS et al. Degenerative disc disease: assessment of changes in vertebral body marrow with MR imaging. Radiology 1988; 166:193199.

2. Stimac G, Porter BA, Olson DO et al. GadoliniumDTPA-enhanced MR imaging of spinal neoplasms: Preliminary investigation and comparison with unenhanced spin-echo and STIR sequence. $A m J$ Roentgenol 1988; 151:1185-1192.

3. Yu S, Rosenbaum AE, Poe LB et al. The lumbar spine. In Chan WP, Lang P, Genant HK (eds). MRI of the musculoskeletal system. WB Saunders, Philadelphia, 1994 pp 156-157.

4. Frank JA, Ling A, Patronas NJ et al. Detection of malignant bone tumors: MR imaging vs. scintigraphy. Am J Roentgenol 1990; 155:1043.

5. Schmorl G, Junghanns H (eds). The Human Spine in Health and Disease. Grune \& Stratton, New York, 1959, p 12.
6. Laredo JD, ReizineD, Bard M et al. Vertebral hemangiomas: Radiologic evaluation. Radiology 1986; 161:183.

7. Mohan V, Gupta SK, Tuli SM. Symptomatic vertebral hemangiomas. Clin Radiol 1980; 31:575.

8. Kransdorf MJ, Stull MA, Gilkey FW et al. Osteoid osteoma: Radiographics 1991; 11:671.

9. Jackson RP, Reckling FW, Mantz FA. Osteoid osteoma and osteoblastoma. Similar histologic lesions with different natural histories. Clin Orthop 1977; 128:303-313.

10. Ricci C, Cova M, Kang YS et al. Normal age related patterns of cellular and fatty bone marrow distribution in the axial skeleton: MR imaging study. Radiology 1990; 77:83.

11. Moore SG. Pediatric marrow and musculoskeletal MRI. In Hasso AN, Stark DD (eds). Spine and Body Magnetic Resonance Imaging. Categorical Course Syllabus, American Roentgen Ray Society, Boston, 1991 p 145.

12. Unger EC, Summers TB. Bone Marrow Topics. Magn Reson Imaging 1989; 1:31.

13. Vogler JB, Murphy WA. Bone Marrow Imaging. Radiology 1988; 168:679.

14. deRoos A, van Persijn van Meerten EL, Bloem JL et al. MRI of tuberculous spondylitis. Am J Roentgenol 1986; 147:79-82.

15. Modic MT, Feiglin DH, Piriano DW et al. Vertebral osteomyelitis: Assessment using MR. Radiology 1985; 157:157-166.

16. Thrush A, Enzmann D. MR imaging of infectious spondylitis. Am J Roentgenol 1990; 11:1171.

17. Slipman CW, Shin CH, Patel RK et al. Etiologies of the failed back surgery syndrome. Pain Medicine 2002; 3: in press.

18. Ross JS, Delamarter R, Hueftle MG et al. Gadolinium-DTPA-enhanced MR imaging of the lumbar spine: Time course and mechanism of enhancement. Am J Roentgenol 1989; 152:825-834.

19. Hueftle M, Modic MT, Ross JS et al. Lumbar spine: Post-operative MR imaging with Gd-DPTA. Radiology 1988; 167:817. 